\section{Integration of micro-services as components in modeling environments for low code development}

1,2H.A.A. Chaudhary, ORCID: 0000-0001-9272-2622<ahmad.chaudhary@ul.ie>

${ }^{1,3}$ T. Margaria, ORCID: 0000-0002-5547-9739<Tiziana.Margaria@ul.ieg>

${ }^{1}$ University of Limerick,

Limerick, V94 T9PX, Ireland
Liversity of Limerick,

${ }^{2}$ Confirm Research Centre for Smart Manufacturing

IBC Block2, University of Limerick, Limerick, Ireland

${ }^{3}$ Lero: The Irish Software Research Centre

Tierney Building, University of Limerick, Ireland

Abstract. Low code development environments are gaining attention due to their potential as a development paradigm for very large scale adoption in the future IT. In this paper, we propose a method to extend the (application) Domain Specific Languages supported by two low code development environments based on formal models, namely DIME (native Java) and Pyro (native Python), to include functionalities hosted on for heteroger integration, both ext enterprise systems survices, in our case Amazon Web Services (AWS) (but this can be easily generalized to other cloud platforms). Developers can this way utilize within DIME and Pyro the potential of sophisticated services, potentially the entire Python and AWS ecosystems, as libraries of drag and drop components in thei model driven, low-code style. The new DSLs are made available in DIME and Pyro as collections of implemented SIBs and blocks. Due to the specific capabilities and checks underlying the DIME and Pyro platforms, the individual DSL functionalities are automatically validated for semantic and syntactical errors in both environments.

Keywords: Domain Specific Language (DSL); Model Driven Development (MDD); eXtreme Model Driven Development (XMDD); Service Independent Building Blocks (SIBs); Low code development environments; DIME; Pyro

For citation: Chaudhary H.A.A., Margaria T. Integration of micro-services as components in modeling environments for low code development. Trudy ISP RAN/Proc. ISP RAS, vol. 33, issue 4, 2021, pp. 19-30. DOI: 10.15514/ISPRAS-2021-33(4)-2

Acknowledgements. This work was supported by the Science Foundation Ireland grants 13/RC/2094 (Lero, the Irish Software Research Centre) and 16/RC/3918 (Confirm, the Smart Manufacturing Research Centre).
1,2 X.A.A. Чaydxapu, ORCID: 0000-0001-9272-2622<ahmad.chaudhary@ul.ie>

1,3 T. Маргария, ORCID: 0000-0002-5547-9739 <Tiziana.Margaria@ul.ieg>

\author{
${ }_{1}$ Университет Лимерика,
}

Ирландия, V94 Т9PХ, Лимерик

${ }^{2}$ Confirm: Исследовательский центр интеллектуального производства Ирландия, Лимерикский университет, IBC Block2

${ }^{3}$ Lего: Ирландский центр исследований программного обеспечения Ирландия, Лимерикский университет, Здание Тирни

Аннотация. Среды разработки с низким кодом привлекают внимание из-за их потенциала в качестве парадигмы разработки для очень крупномасштабного внедрения в ИТ будущего. В этой статье мы предлагаем метод расширения (приложений) предметно-ориентированных языков, поддерживаемых двумя средами разработки с низким уровнем кода, основанными на формальных моделях, а именно DIME (родная Java) и Pуro (родной Руthon), для включения функций, размещенных на гетерогенных технологиях и платформы. Для этого мы следуем аналогии с микросервисами. После этой интеграции обе среды могут использовать связь с уже существующими удаленными службами RESTful и корпоративных систем, в нашем случае Amazon Web Services (AWS) (но это можно легко распространить на другие облачные платформы). Таким образом, разработчики могут использовать в DIME и Pуго потенциал спожных сервисов, потенциально всей экосистемы Python и AWS, в виде библиотек перетаскиваемых компонентов в управляео ими стиле с низким кодом. Новые DSL DIME и Pyro aк кол Руго

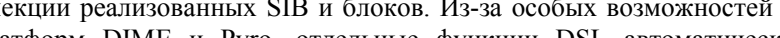
проверяются на семантические и синтаксические ошибки в обеих средах.

Ключевые слова: предметно-ориентированный язык (DSL); управляемая моделями разработка (MDD); экстремальная управляемая моделями разработка (XMDD); сервисно-независимые компоненти (SIB); среды малокодовой разработки; DIME; Piro

Для цитирования: Чаудхари Х.А.А., Маргария Т. Интеграция микросервисов как компонентов сред моделирования для малокодовой разработки. Труды ИСП РАН, том 33, вып. 4, 2021 г., стр. 19-30 (на английском языке). DOI: 10.15514/ISPRAS-2021-33(4)-2

Благодарности: Эта работа была поддержана грантами Ирландского научного фонда 13/RC/2094 (Lero, Ирландский исследовательский центр программного обеспечения) и 16/RC/3918 (Confirm, Исследовательский центр интеллектуального производства).

\section{Introduction}

Low code development platforms enable their users to design and develop applications with minima coding knowledge [1], with the support of drag-and-drop visual interfaces that operate on representations of code as encapsulated code wrappers. The main aim [2] of these platforms is to produce flexible, cost effective and rapid applications in a model driven way. Ideally, they are adaptive to enhancements and less complex is terms of maintenance. Model-driven development (MDD) is an approach to develop such systems using models and model refinement from the conceptual modelling phase to the automated model-to-code transformation of these models to executable code [3]. The main challenges with traditional software development approaches are the complexity in development at large scale, the maintenance over time, and the adaptation to dynamic requirements and upgrades [1]. Doing this on source code is costly, and it systematically excludes the application domain experts. who are the main knowledge and responsibility carriers. At the same time, the cost of quality documentation and training of new human resources for code-based development are other concerns in companies and organizations that depend on code. 
Domain Specific Languages (DSLs) conveniently encapsulate most complexities of the underlying application domain. Encapsulation of code and abstraction to semantically faithful representations in models empowers domain experts to take advantage of these platforms. They can develop products in an efficient manner and also meet the growing demands of application development without having deep expertise in software development. Based on a study [4] from 451 researches, the maintenance effort with low code platforms proved to be $50-90 \%$ more efficient as compared to changes with classical coding languages.

Software systems in general, and especially web apps in internet-centered ecosystems and digital threads in an Industry 4.0 context, are not isolated in nature: they demand interaction with various external systems, libraries and services. Frequent needs are (but not limited to)

- acquire sensors data from external systems,

- feed data to external dashboards for analytics and publishing,

- utilize the compute power of cloud systems,

- sophisticated enterprise services.

In this context, microservices [5] play an important role at the enterprise level. The microservices paradigm (SOA done right) defines certain methods to design software services as suite of independently deployable components with the purpose of modularity, reusability and autonomy [5]. Different versions of these services may coexist in a system as a set of loosely coupled collaborative components and must be independently replaceable without impacting the operations of heterogeneous systems.

This paper proposes the integration of microservices as components in two graphical modelling development environments based on formal models: the general purpose, desktop DIME [6] Integrated Modelling Environment and the special purpose, web based Pyrus (Pyro) [7]. Their extension and integration with external systems through services extends the capabilities of these platform to meet wider communication needs (e.g. in the cloud), and also to take advantage of existing sophisticated enterprise services (e.g. AWS).

Low-code programming both at the API and the platform level is considered to be a game changer for the economy of application development. Gartner Inc., for example, predicts [8] that the size of the low-code development tools market will increase by nearly $30 \%$ year on year from 2020 to 2021 , reaching a $\$ 5.8$ billion value in 2021 . They state that so far, this is the fastest and probably the simplest and most economical method of developing applications.

In this paper, Section 2 discusses the state of art, Section [3] states the problem, Section [4] gives an overview of the platforms used to extend the low-code DSLs. Section [5] explains the integration, architecture and implementation of SIBs in DIME (the desktop IME) and blocks in Pyro/Pyrus (the web IME). Finally, in Section 6 we conclude and discuss.

\section{State of the art}

Most domain specific languages today are at the coding level and do not leverage a model driven approach at the platform level. The rise in re-usability and maintainability demands paved the path to low code development environments and gained the attention of the developer's community [9] The construction of meta-models behind these DSLs is challenging, since they must capture all the domain knowledge, i.e. provide both semantic and syntactic rules.

Ktrain [10] is a popular coding level DSL: a python wrapper that encapsulates Tensor Flow functionalities and facilitates developers to augment machine learning tasks with fewer lines of python code. Xatkit [11], still in early stages of development, increases the reusability of chat bots by evolving NLP/NLU engine for text analytics. At the language level they support several versions of bots, but the generation of chatbots from existing data sources at the framework level is in future plans. jABC [12] is a general purpose XMDD framework for the development of desktop and enterprise applications in model driven fashion. It enables its users to compose models by drag and drop of reusable blocks into hierarchical graph structures that are executable (interpreted) and compilable. Aurera [1] is a standalone desktop system for business modelling and addresses the challenges of frequent changes to IT solutions. The system is in early stages of development and does not support communication with external systems.

DIME [6] is a general purpose MDD platform-level tool, suitable for agile development due to its rapid prototyping for web application development. It follows the One Thing Approach based on XMDD [13], in a lineage of development environments that traces back to the METAFrame' 95 [14] DIME supports both control flow and data flow modelling in its process diagrams. Control flow models admit a single start node but may have multiple end nodes, and nodes (called SIBs) representing single functionalities or sub-models are graphs, i.e. formal models. The SIBs are connected via directed edges depending on the business logic, with distinct edge types for dataflow and control-flow.

Agent-based modelling paradigm [15] is another popular approach to increase the development productivity in simulation environments. CaaSSET [16] is a Context-as-a-Service based framework to ease the development of context services. The transformation into executable services is semiautomatic.

The market segment of web based development environments is still relatively young. Not having many established environments, there is a huge potential for research and collaboration in this area Theia [17], is a textual DSL tool supporting both desktop and web based IDEs.

Pyro [7] is a web base graphical modelling environment for the collaborative development of web applications based on DSLs. Pyro, like DIME, is itself a product modelled with the Cinco [18] Meta Tooling Framework, which provides a suite of textual DSLs in which to specify the models for which to generate editors. The MGL («Meta Graph Language») defines the structural information on the tool's model; the «Meta Style Language» (MSL) file specifies the visual characteristics (e.g. shapes and colors) of this model. The «Cinco Product Definition» (CPD) file specifies the details of the tool generation.

Both DIME and Pyro are advanced graph model editors generated in this way from Cinco specifications. In this sense, they share a common philosophy, the semantic and syntactic characteristics of their respective models and edit/check/manipulate capabilities are described formally in their MGL, MSL and CPD files.

To interact with external entities, Micro service [19] is a popular way to develop modular, reusable and autonomous service components. We adopt this approach to extend the functionalities of two of the platforms in a model driven way. Following the same principles of graphical microservices architectures, AjiL [20] is a good effort in this direction, but due to performance delays in complex applications, they shifted their focus from graphical to textual notations.

\section{Problem Statement}

We consider the DIME [6] and Pyro [7] Cinco-products as our case study. Both are graphica Integrated Modelling Environments for low-code/no-code application development, used to develop research $[21,22]$ as well as industrial applications. We will use DSLs to virtualize the technological heterogeneity of the services, delivering a simple, coherent and efficient extension to both low-code modelling platforms.

Concretely, we show how to extend the capabilities of the DSLs through new, heterogeneous services. We

1) extend DIME, an offline eclipse-based general-purpose MDD environment for Web applications, by integrating a generic RESTful service as a new component, technically adding a new executable SIB that a) represents and b) executes this REST service;

2) extend Pyrus/Pyro, a collaborative, web based special-purpose MDD environment for data analytics and $\mathrm{AI} / \mathrm{ML}$, by integrating cloud-based enterprise services in a similar fashion. Here we chose Amazon Web Services. 
The models in the 2 IMEs are different: DIME has rich models that cover processes, data, GUI, roles and security, and supports both dataflow and control flow models. Pyrus is simpler, and supports only dataflow modelling, which is popular and sufficient in the analytics pipelines it addresses. As the specific integration depends on the characteristic and expressive power of the models, there are differences.

The extension by integration adds to the tools the capability to communicate with sophisticated enterprise ecosystems, without sacrificing the flexible yet intuitive modelling style for the no-code users, who just use the DSLs that are available.

\section{Overview of the IMEs}

Domain-specific languages aim at minimizing the domain/IT knowledge gap between domain experts and software developers by lifting the vocabulary, granularity and structure of the application domain into the modelling language, so that the modelling entities stay familiar to the domain experts and their intuition is indeed correct. Domain experts prefer graphical languages because of the haptic functionality of drag-and-drop from a collection of functionalities is an apt metaphor for the construction of complex behaviors from an appropriate network of identifiable, well understood building blocks along intuitive control flow and data flow patterns.

The effort to develop these tools from scratch is enormous. Consequently, the specialization and evolution of such tools is hindered by the sheer cost and complexity of managing their code and its quality and support. Cinco [18] was a game changer: a meta-level platform that wipes out this cost and complexity by providing the above described domain specific graphical modelling and code generation capabilities. Most Cinco products are based on Eclipse, enhanced with graphical modelling tools and various plug-ins. Suddenly, one can create a new Integrated Modelling Environment by specifying properties in three files and availing of the Cinco code generation capability for the target execution environment (e.g., eclipse or web). Modifications are not anymore at the code level: to change DIME or Pyro, one edits the specifying files and re-generates the tool with the appropriate generator.

In this paper ${ }^{1}$, we will discuss the extension and integration of external systems as micro services in two of the Cinco's products DIME and Pyro (particularly Pyrus).

\subsection{DIME}

DIME is an Integrated Modeling Environment based on J2EE eclipse, to design, develop and deploy web applications in an agile paradigm. Its model types help users to graphically model and develop different aspects of ordinary web application: (i) data model, (ii) GUI model, (iii) business logic in terms of processes and persistence, and (iv) roles and security model. The specific functional capabilities are provided to the users as a family of Graphical DSLs. The GUI DSL and a DSL providing a collection of generic blocks (called SIBs, for Service Independent Building Blocks) come with DIME, and other, domain specific DSLs can be added at need.

Modelling in DIME happens mainly by the mechanism of drag-and-drop of DSL components on a canvas, and components comprise a node and a predefined set of outgoing edges. DIME supports both data and control flow to implement different aspects of business logic. Consistency checks are built-in in the DIME MGL and MSL, so that errors are either prevented (e.g., an output cannot connect to another output) or detected (e.g. the model is incomplete because some edges are dangling, not connected). DIME follows the One Thing Approach philosophy [23] by enforcing the SIBs to be generic and encapsulate only the required functionality. This way, SIBs are easily understandable and reusable, and application experts that are not coders can develop complex applications by using the SIBs in the provided DSLs. GUI models represent single pages of the web application and links to the underlying functionalities. Process models can be hierarchical, i.e. contain other process models, this way easing the organization and comprehension of the structure contain other process models, this way easing the organization and comprehension of the structure
and behaviour of complex applications. Once the models are ready, the product generation step feeds the models collection to successive model-to-model and model-to-code transformers, resulting in a complete code generation for a standard web application runtime.

The setup for the development environment for DIME requires Java version 1.8 and eclipse dependencies to be installed on the development machine.

\subsection{Pyro}

In contrast to DIME, Pyro is a web based Cinco-product that runs in a web browser and turns it into a collaborative domain-specific graphical modelling environment for data-flow applications.

\%The high level metamodeling is braced by MGL and MSL: The MGL depicts the syntactical constraints including the available nodes and edges; and the visual appearance of these modelling artefacts is defined in MSL. Pyro stores objects and data types in a loosely coupled manner [24]. To incorporate the rich features of typical web application, like the built-in support of cross-platform and a reusable components focused architecture, its front-end is built upon the Angular Dart [25] framework. To meet the needs of uninterruptible user interaction with the modelling environment, data exchange is implemented via non-blocking REST-based asynchronous communication. As a more recent development, Pyro is being enhanced with performance optimization and integration of external systems.

Pyrus is a specific Pyro derivative specialized for dataflow models executing within the popular Jupyter notebook environment. It is therefore particularly attractive for data analytics and AI applications, that are frequently coded in Python.

Working with Pyro/Pyrus requires the platform deployment on a local or remote server accessible via browser.

\section{Proposed NPU architecture modifications}

\subsection{Memory synchronization via the shared bus}

In this approach, the following modifications are proposed: memory devices containing the algorithm state are added to each pipeline stage. Memory devices on pipeline stages with equal depths are connected using a shared bus. The bus is used to synchronize data in the memory devices. When a memory cell in some memory device is updated, the new value and memory cell address are sent to other memory devices via the bus.

There are two operations available for the processing unit: read a value from a memory cell and write a value to a memory cell. When the value is read it is taken from the memory device that is on the same pipeline stage. When the value is written to the memory it is written to the memory device on the same pipeline stages and then is sent to appropriate memory devices in other pipelines.

\subsection{Combining mechanism}

In this approach, memory devices and processing units are connected using a packet switched network-on-chip with special switches that allow to combine memory requests. Memory request have a Read-Modify-Write form. Memory request has a form of a tuple (id, addr, $f$ ) where $i d$ is a unique request identifier, $a d d r$ is a memory cell address and $f$ is a memory operation identifier. The response consists of two values: request identifier and the value that was in the memory cell before the memory operation was done.

This approach requires memory cells and network switches to have specialized arithmetic units to perform memory operations and memory request combinations. 


\section{Extending the IMEs}

We show now how to extend DIME and Pyrus with RESTful services and cloud-based AWS services, respectively. This happens by implementing a new DSL consisting of a collection of capabilities that run on an external platform in a different technology. Effectively, these are akin to microservices. We show here exemplarily how to implement one such microservice for each case. The extension to other RESTful services or other AWS or similar services is then easy to achieve following these blueprints.

\subsection{RESTful extension of DIME}

We show now how to develop a generic Service Independent Building Block (SIB) in DIME that communicates with any external RESTful system.

Extending the DIME functionality happens by using the support of native library it provides. In DIME's multi-model type architecture, the business/logic model type is where the new SIBs will be utilized. As shown in fig. 1, the existing model architecture is extended with the addition of a native library as a new block belonging to the process/business logic model type. The native block will be merged to the process/business logic models during the automated code generation phase for the web application. Concretely, the extended functionality will be integrated as Java code with the remainder of the application during the compilation, and this way it will not add any additional performance penalty.

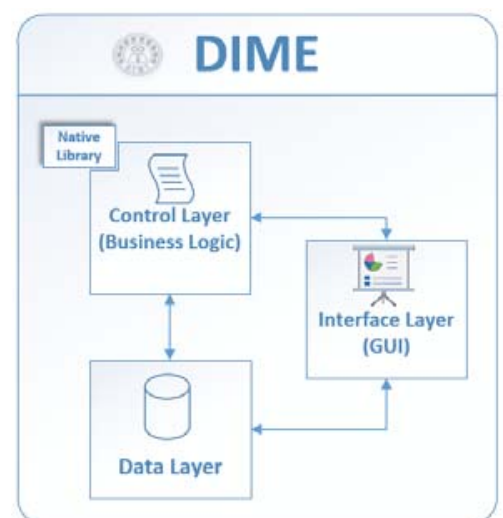

Fig. 1. DIME: Modelling Architecture and Native Library support

For the SIB implementation, we consider here a REST service that acts as a server and returns a list of country names on the basis of a country code input, e.g. United Kingdom for input 'uk', and the name of all countries from the database for input 'all'. The service is implemented in PHP in a conventional fashion, and deployed on an external public server. It will respond to the requests generated by client SIBs

Now, we need to create a new client SIB with appropriate characteristics to communicate with RESTful service. This encompasses the SIB declaration and the SIB implementation.

The SIB declaration is shown in Listing 1.

package app.demo

sib rest_read_str_list : file_path\#Java_fn

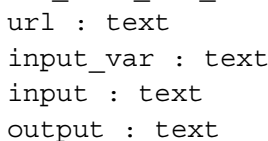

\section{output: [text] \\ $>$ noresult \\ -> failure \\ Listing 1. SIB declaration for the «REST Read» SIB}

- Firstly, in the project explorer we add a new, empty file with extension «.sib» and the name of the proposed SIB.

- This SIB declaration file contains the signature of the new SIB. It starts with the keyword «sib», followed by the new SIB name, that in our case is REST read str list, followed by a colon and the path to the attached Java function. This is the function be invoked when the SIB is used in the process modelling.

- The next section contains the proper signature: the list of inputs and outputs, with name and data types. In our case, the SIB accepts the following I/O:

- URL of an external server;

- input variable name and data to create a valid URL at run time;

- the output variable name is also added in the signature, to extract the requested data from server response for further JSON parsing.

- Finally, the list of different control branches based on outcomes. In our case the three branches are «success», which returns a text output provided by the external service, «noresult» of the external services returns no result, and «failure» in case of error in the communication with the external service.

For the SIB implementation, The RESTful «Rest Read» service is implemented in PHP in a conventional fashion, and deployed on an external public server. It will respond to the requests generated by this SIBs.

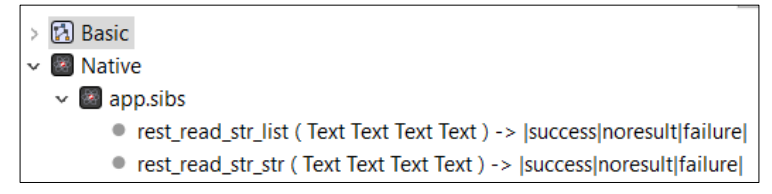

Fig. 2. SIBs explorer with the new Native SIBS

Once the declared SIB, its signatures and the attached Java function are validated by the platform, the SIB will be visible in the explorer as a Native SIB, with the other default SIBs as shown in fig. 2. At this point it is ready to be used, and available to the DIME users as a drag and drop item, ready to be inserted in any process model.

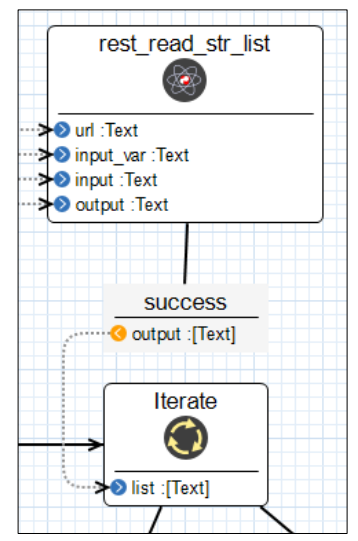

Fig. 3. The REST Read SIB in use: Visual representation in a model 
Fig. 3 shows the visual representation of the newly developed SIB, as it appears when it is used in a process model. The required four inputs are being fed to this block using data flow (dotted) arrows. We see the three outgoing branches, labelled as defined. On success, the result will be conveyed as a string (or list of strings) to the successive SIB.

DIME automatically validates semantic and syntactic errors after the insertion and data connectivity of SIBs, ensuring this way the correctness of intended behaviour (automatic quality assurance [26] of models).

\subsection{Cloud extension of Pyrus}

We extend now the Pyrus is an online data analytics platform built using Pyro with Amazon Web Services (AWS), choosing the Amazon Translate service [27].

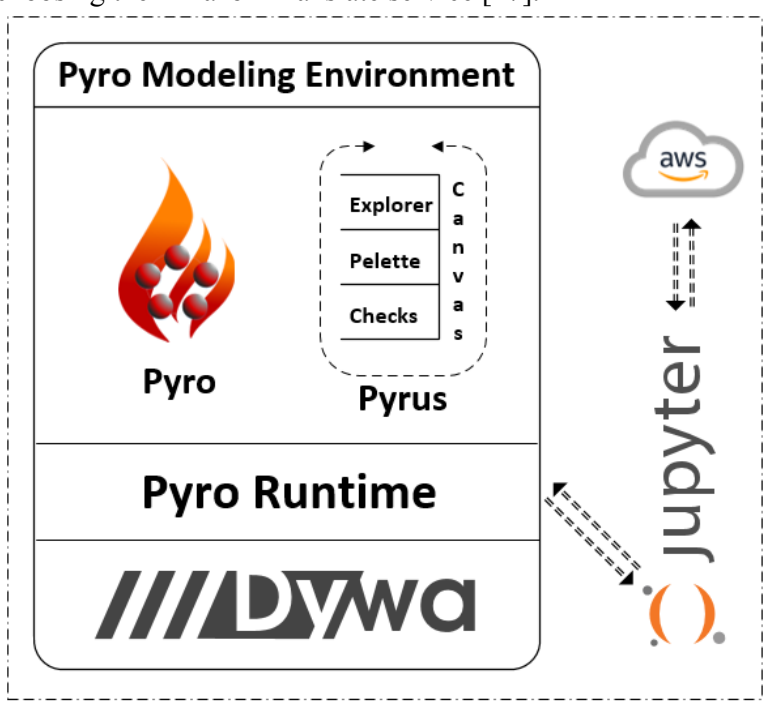

Fig. 4. The Pyrus/Pyro Architecture extended with AWS

Pyrus communicates with Jupyter hub at the backend. It uses the RESTful protocol to read function signatures and execute the attached python code. As shown in fig. 4, Jupyter and Pyrus communicates in asynchronously manner.

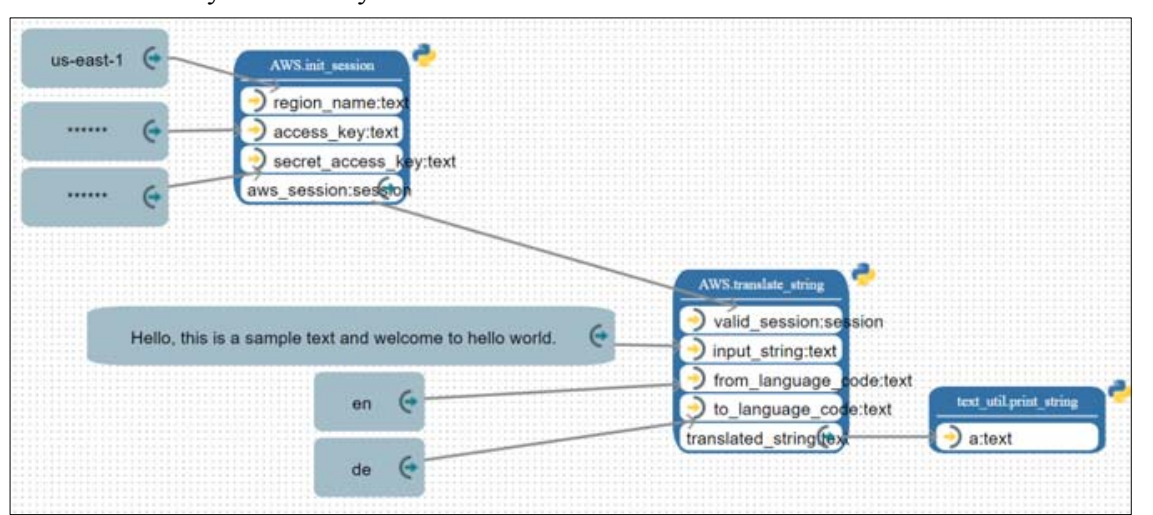

Fig. 5. Pyrus pipeline using AWS translate
The mechanism for the new AWS Translate block definition and implementation is similar to the The mechanism for the new AWS Translate block definition and implementation is similar to the
DIME SIB declaration, but it only contains the signature, no outgoing branches. As Pyrus supports a dataflow modelling style, there are no control elements (the branches). The signature declaration starts with the \# keyword, it is followed by meta data and the implementation of the functions in a python file. It has an extension «.py» and is located in the Jupyterhub space. Pyrus automatically reads these annotated signatures and shows them as drag-able blocks in its explorer.

Fig. 5 shows the working pipeline of AWS_translate: in reality we have defined 2 blocks, AWS.init_session and AWS.translate_string.

The initialization block must meet the preconditions of the external server in order to use its services. Communication with the AWS server/services requires a valid session, validated with credentials, i.e. access key, secret key, server information. The AWS.init session initiates the communication transaction with the AWS server. It accepts the required inputs/tokens from connected grey blocks, which are constants.

Once successfully authenticated by AWS, a session token is provided for further communication with AWS. This token (output) is fed to the AWS translate string block along with the other require inputs: the text string to be translated and the code of the from and to languages.

Finally, the (translated text) result is passed to the next block, text_util.print_string, that prints it on screen.

The pipelines are automatically validated by the underlying modelling platform to check for connectivity errors of the blocks on the canvas.

\subsection{Tool and Technologies}

The tools and technologies used for these implementations and extensions are Eclipse, Java, JSON library, PHP, Python, DIME, Pyrus, Jupyter Hub and Amazon Web Services.

As the methodology is generic, it can be followed like a blueprint to implement communication and integrate a large variety of external services and platforms. The resulting drag and drop components enrich the DSL domain and expressive features of low code development in the mentioned platforms.

\section{Conclusion and Discussion}

We presented a generic extension mechanism to two low code development environments along a microservice philosophy. We showed it by integrating preexisting remote RESTful services and cloud-based enterprise system services as new drag and drop components in the respective DSLs. In DIME, an offline low-code IME, we used the native library mechanism, with signature declaration, linked Java backend code, and the code is merged with the logic layer at compile time. Pyrus, an online no code graphical data analytics tool, is linked with Jupiter Hub for functions discovery and code execution. To display new python functions as components in Pyrus, custom signatures are added to the python files defined in Jupyter hub, and the data flow pipeline of the service is modelled in the Pyrus frontend.

The simplicity and generality of the integration are an important feature of the chosen platforms. We envisage in fact a systematic integration of DSLs for various application domains stemming for our research collaborations. The simpler this is, the easier is the adoption of the approach across diverse application domains, research groups, and industrial partners. The (hand)code based extension approach of most popular low-code environments, that do not use formal models, nor generate «intelligent» modelling domains that have built-in checks for the model conformance are in fact inferior and sources again of complexity in the management of heterogeneity, code maintenance and evolution. The next application domains will be data visualization and data streaming platforms. We will support more AI/ML and data analytics functionality both in DIME and in Pyrus, adding 
also cross-platform integration, in order to use the analytics capabilities of Pyrus pipelines in the DIME Digital Twin applications for Industry 4.0 as well as in the Digital Humanities [28]

\section{References}

[1] R. Waszkowski. Low-code platform for automating business processes in manufacturing. IFACPapersOnLine, vol. 52, issue 10, 2019, pp. 376-381.

[2] R. Sanchis, Ó. García-Perales et al. Low-code as enabler of digital transformation in manufacturing industry. Applied Sciences, vol. 10, no. 1, 2020, $17 \mathrm{p}$.

[3] S.J. Mellor, T. Clark, and T. Futagami. Model-driven development: guest editors' introduction. IEEE Software, vol. 20 , no. 5 , 2003, pp. 14-18.

[4] Intelligent process automation and the emergence of digital automation platforms. Available at: https://www.redhat.com/cms/managed-files/mi-451-research-intelligent-process-automation-analystpaper-f11434-201802.pdf, accessed Feb, 2021

[5] S. Newman. Building microservices: designing fine-grained systems. O'Reilly Media, 2015, $280 \mathrm{p}$.

[6] S. Boßelmann, M. Frohme et al. Dime: A programming-less modeling environment for web applications Lecture Notes in Computer Science, vol. 9953, 2016, pp. 809-832.

[7] P. Zweihoff, S. Naujokat, and B. Steffen. Pyro: Generating domain-specific collaborative online modeling environment. Lecture Notes in Computer Science, vol. 11424, 2019, pp. 101-115.

[8] Gartner forecasts. Available at: https://www.gartner.com/en/newsroom/press-releases/2021-02-15 gartner-forecasts-worldwide-low-code-development-technologies-market-to-grow-23-percent-in-2021, accessed Feb, 2021

[9] K. Ordoñez, J. Hilera, and S. Cueva. Model-driven development of accessible software: a systematic literature review. Universal Access in the Information Society, 2020, pp. 1-30.

[10] A.S. Maiya. ktrain: A low-code library for augmented machine learning. arXiv preprint arXiv:2004.10703, $2020,9 \mathrm{p}$

[11] G. Daniel, J. Cabot et al. Xatkit: A multimodal low-code chatbot development framework. IEEE Access, vol. 8, 2020, pp. $15332-15346$

[12] B. Steffen, T. Margaria et al. Model-driven development with the jabc. Lecture Notes in Computer Science, vol. 4383, 2007, pp. 92-108.

[13] T. Margaria and B. Steffen. eXtreme Model-Driven Development (XMDD) Technologies as a Hands-On Approach to Software Development Without Coding. In Tatnall A. (eds) Encyclopedia of Education and Information Technologies. Springer, 2020.

[14] B. Steffen, T. Margaria et al. The metaframe' 95 environment. Lecture Notes in Computer Science, vol. 1102, 1996, pp. 450-453

[15] F. Santos, I. Nunes, and A. L. Bazzan. Quantitatively assessing the benefits of model-driven developmen in agent-based modeling and simulation. Simulation Modelling Practice and Theory, vol. 104, 2020, article no. 102126.

[16] H. Moradi, B. Zamani, and K. Zamanifar. Caasset: A framework for model-driven development of context as a service. Future Generation Computer Systems, vol. 105, 2020, pp. 61-95.

[17] Cloud and desktop ide platform. Available at: https://theia-ide.org/, accessed Feb, 2021

8] S. Naujokat, M. Lybecait et al. Cinco: a simplicity-driven approach to full generation of domain-specific graphical modeling tools. International Journal on Software Tools for Technology Transfer, vol. 20, issue 3 , pp. 1-28, 2018.

[19] L. Baresi and M. Garriga. Microservices: The evolution and extinction of web services? In Microservices: Science and Engineering, Springer, 2020, pp. 3-28

[20] F. Rademacher, J. Sorgalla et al. Graphical and textual model-driven microservice development. In Microservices: Science and Engineering, Springer, 2020, pp. 147-179.

[21] T. Margaria and A. Schieweck. The digital thread in industry 4.0. Lecture Notes in Computer Science, vol. 11918, 2019, pp. 3-24.

[22] S. Jorges, C. Kubczak et al. Model driven design of reliable robot control programs using the jabc. In Proc. of the Fourth IEEE International Workshop on Engineering of Autonomic and Autonomous Systems (EASe'07), 2007, pp. 137-148.

[23] T. Margaria and B. Steffen. Business process modeling in the jabc: the one-thing approach. In Handbook of research on business process modeling. IGI Global, 2009, pp. 1-26.

[24] J. Neubauer, M. Frohme et al. Prototype driven development of web applications with dywa. Lecture Notes in Computer Science, vol. 8802, 2014, pp. 56-72.
[25] Angular dart open source packages. Available at: https://github.com/angulardart, accessed Feb, 2021.

[26] S. Windmüller, J. Neubauer et al. Active continuous quality control. In Proc. of the 16th International ACM SIGSOFT Symposium on Component-based Software Engineering, 2013, pp. 111-120.

27] Amazon translate; fluent and accurate machine translation. Available at: https://aws.amazon.com/translate/, accessed Feb, 2021

[28] Khan R., Schieweck A. et al. Historical Civil Registration Record Transcription Using an eXtreme Mode Driven Approach. Trudy ISP RAN/Proc. ISP RAS, vol. 33, issue 3, 2021, pp. 123-142.

\section{Information about authors / Информация об авторах}

Hafiz Ahmad Awais CHAUDHARY is a PhD student in University of Limerick and associated with Confirm - a SFI research centre in Smart Manufacturing. Ireland. His area of research includes «Interoperability and Data Integrations in the Digital Smart Manufacturing». Before moving to Ireland, he has been working as a Lecturer in University of Engineering and Technology, Lahore, Pakistan.

Хафиз Ахмад Аваис ЧАУДХАРИ - аспирант Лимерикского университета, сотрудничающий c Confirm - исследовательским центром Ирландского научного фонда в области интеллектуального производства. Его область исследований включает «Функциональная совместимость и интеграция данных в интеллектуальном цифровом производстве». До переезда в Ирландию он работал преподавателем в инженерно-технологическом университете в Лахоре, Пакистан.

Tiziana MARGARIA, PhD in Computer and Systems Engineering, Politecnico di Torino, Italy, Professor at Computer Science and Information System Department at the University of Limerick, Ireland. Research Interest: eXtreme Model Driven Design, lightweight formal methods, automatic program synthesis, system correctness, in particular compliance and security, future education in SE and IT.

Тициана МАРГАРИА, кандидат компьютерных наук и системной инженерии, Туринский политехнический университет, Италия, профессор кафедры компьютерных наук информационных систем. Область научных интересов: проектирование на основе экстремальных моделей, упрощенные формальные методы, автоматический синтез программ, правильность системы, в частности соответствие и безопасность, будущее образование в области программной инженерии и информационной технологии. 